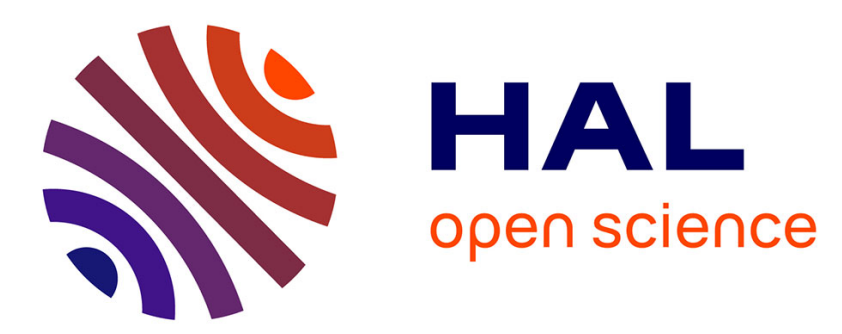

\title{
Voxel-based multi-model fitting method for modelling time activity curves in SPECT images
}

David Sarrut, Adrien Halty, Jean-Noël Badel, Ludovic Ferrer, Manuel Bardiès

\section{To cite this version:}

David Sarrut, Adrien Halty, Jean-Noël Badel, Ludovic Ferrer, Manuel Bardiès. Voxel-based multimodel fitting method for modelling time activity curves in SPECT images. Medical Physics, 2017, 44 (12), pp.6280-6288. 10.1002/mp.12586 . hal-01611266

\section{HAL Id: hal-01611266 https://hal.science/hal-01611266}

Submitted on 4 Apr 2019

HAL is a multi-disciplinary open access archive for the deposit and dissemination of scientific research documents, whether they are published or not. The documents may come from teaching and research institutions in France or abroad, or from public or private research centers.
L'archive ouverte pluridisciplinaire HAL, est destinée au dépôt et à la diffusion de documents scientifiques de niveau recherche, publiés ou non, émanant des établissements d'enseignement et de recherche français ou étrangers, des laboratoires publics ou privés. 


\title{
Voxel-based multimodel fitting method for modeling time activity curves in SPECT images
}

\author{
David Sarrut, ${ }^{\text {a) }}$ Adrien Halty, and Jean-Noel Badel \\ Université de Lyon, CREATIS, CNRS UMR5220, Inserm U1044, INSA-Lyon, Université Lyon 1, Centre Léon Bérard, Lyon, France \\ Ludovic Ferrer \\ Medical Physics Department, ICO René Gauducheau, St Herblain, France \\ CRCINA UMR 892, Nantes, France
}

Manuel Bardiès

Inserm UMR1037, CRCT, Université Toulouse III-Paul Sabatier, F-31000, Toulouse, France

(Received 27 March 2017; revised 22 August 2017; accepted for publication 22 August 2017; published $\mathrm{xx}$ Xxxx Xxxx)

\begin{abstract}
Purpose: Estimating the biodistribution and the pharmacokinetics from time-sequence SPECT images on a per-voxel basis is useful for studying activity nonuniformity or computing absorbed dose distributions by convolution of voxel kernels or Monte-Carlo radiation transport. Current approaches are either region-based, thus assuming uniform activity within the region, or voxel-based but using the same fitting model for all voxels.

Methods: We propose a voxel-based multimodel fitting method (VoMM) that estimates a fitting function for each voxel by automatically selecting the most appropriate model among a predetermined set with Akaike criteria. This approach can be used to compute the time integrated activity (TIA) for all voxels in the image. To control fitting optimization that may fail due to excessive image noise, an approximated version based on trapezoid integration, named restricted method, is also studied. From this comparison, the number of failed fittings within images was estimated and analyzed. Numerical experiments were used to quantify uncertainties and feasibility was demonstrated with real patient data.

Results: Regarding numerical experiments, root mean square errors of TIA obtained with VoMM were similar to those obtained with bi-exponential fitting functions, and were lower $(<5 \%$ vs. $>10 \%$ ) than with single model approaches that consider the same fitting function for all voxels. Failure rates were lower with VoMM and restricted approaches than with single-model methods. On real clinical data, VoMM was able to fit $90 \%$ of the voxels and led to less failed fits than single-model approaches. On regions of interest (ROI) analysis, the difference between ROI-based and voxel-based TIA estimations was low, less than $4 \%$. However, the computation of the mean residence time exhibited larger differences, up to $25 \%$.

Conclusions: The proposed voxel-based multimodel fitting method, VoMM, is feasible on patient data. VoMM leads organ-based TIA estimations similar to conventional ROI-based method. However, for pharmacokinetics analysis, studies of spatial heterogeneity or voxel-based absorbed dose assessment, VoMM could be used preferentially as it prevents model overfitting. (C) 2017 American Association of Physicists in Medicine [https://doi.org/10.1002/mp.12586]
\end{abstract}

Key words: dosimetry, SPECT, targeted radionuclide therapy, time activity curve, voxel-based

\section{INTRODUCTION}

In targeted radionuclide therapy, the quantitative estimation of the biodistribution and pharmacokinetic of the injected radiolabeled molecular agent is essential for understanding and optimizing treatments. Indeed, it is important for relating physical parameters to biological outcomes (response assessment) or estimating absorbed dose-effect relationship (efficacy or toxicity). ${ }^{1}$ Absorbed dose distributions are most often computed according to the MIRD formalism. ${ }^{2}$ For that purpose, the activity distributions are derived from SPECT or PET images from time-delayed acquisitions as voxel values contain an estimate of activity concentration.

Most of the time, this analysis is based on the definition of regions of interest (ROI) at the organ level. From a delineated
ROI, voxel values inside a ROI are averaged. Thus, a single time activity curve (TAC) per ROI can be estimated from the images acquired at different time-points. Then, time integrated activities (TIA), in Bq.s, are estimated from each TAC by fitting with mono- or multiexponential functions. Absorbed doses are then calculated from TIA by using different approaches based on precomputed S-value tables or explicit radiation transport modeling. However, the analysis is limited to identifiable ROI, prone to contour uncertainties and does not allow for the investigation of activity nonuniformity or heterogeneity, which may be important. ${ }^{3,4}$

To surpass this limitation, several authors have proposed estimating the TIA at the voxel level, performing model fitting and numerical integration for each voxel ${ }^{5-7}$ (among others). Subsequently, voxel-based TIA is used to compute 
an absorbed dose map by convolution of voxel S-values ${ }^{8}$ or Monte-Carlo simulations. ${ }^{9-11}$ In addition to the calibration of input images, which is in itself a difficult task due to several degrading factors (attenuation, scatter, partial volume effect, and noise), voxel-based quantitation requires spatial alignment of all images in the input sequence. Spatial alignment can be performed via deformable image registration, as shown, for example, in Ref. [12-14] A CT image is chosen as reference and other $\mathrm{CT}$ images are aligned to that image. The corresponding deformation vector fields (DVF) are applied to deform all SPECT images in the reference image coordinate system. Once this step is completed, the voxels with the same coordinates in all images are considered to represent the same volume at different time points. The uncertainty associated with this procedure is mostly limited by the SPECT voxel size (around $4 \mathrm{~mm}$ ) which is usually larger than that of the CT (around $1 \mathrm{~mm}$ ). Breathing motion is another limitation leading to image artifacts, such as duplicated or blurred structures, depending on the acquisition speed. ${ }^{10}$ This is mostly important in the chest region where movements are the largest, particularly near the diaphragm.

Once SPECT images are aligned, TAC analysis is performed at voxel level. For example, Jackson et al. ${ }^{5}$ were among the first to propose a computational workflow, named VRAK, to automatize the fitting of a tri-exponential model to every voxel TAC. The authors used an a priori model adapted to ${ }^{177} \mathrm{Lu}$-octreotate peptide receptor radionuclide therapy. Due to a potentially high level of noise in the images, voxel-byvoxel fitting could be a challenge and the authors included preand postconditional procedures to improve fitting parameters determination. Because of the lack of reference, it was not possible to evaluate the quality of the estimated integrated activity.

In clinical cases, pharmacokinetics may vary from one voxel to another and it is unlikely that all voxels can be fitted by a single model. Moreover, fixing an a priori fitting model to a generally unknown voxel-time behavior could lead to errors. Another common issue is the low number of timepoints available in TAC (generally from 3 to 6 ) and attempting to fit with a model with too many parameters is known to lead to overfitting. A method that independently adapts voxel-byvoxel fitting methods is therefore proposed in this study.

\section{METHOD}

In this section, we describe a method that estimates a fitting function for each voxel by automatically selecting the most appropriate model among a predetermined set with Akaike criteria. The method is called voxel-based multimodel (VoMM).

\section{A. Voxel-by-voxel multimodel fitting (VoMM)}

It was assumed that the analyzed SPECT images were calibrated, that is, voxel values were expressed in becquerel (Bq). The images were considered as registered, in such a way that they form a $3 \mathrm{D}+\mathrm{t}$ sequence of activity image $A(x, t)$, with $x$ the voxel coordinates, $t$ the time, $t_{l}$ the time coordinate with indices $l \in[1 \ldots N]$ and $N$ the number of time-points. At any voxel position, $x$, a TAC $A(x, t)$ was thus available. In this study, a set of fitting models derived from a sum of exponentials was used for TAC approximation. Due to the generally low number of time-points $\mathrm{N}$ (typically $N \leq 6$ ), the models with the maximum number of parameters that we use were a sum of two exponentials. Notations similar to those in Kletting et al. ${ }^{15,16}$ were used. We considered the models $f_{2}, f_{3}, f_{4 a}$, $f_{4}$, as shown in Eq. (1). Note that the model $f_{1}(t)=C_{1} e^{-\lambda_{\text {physt }} t}$ was not considered here. $K$ was taken as the number of model parameters. The radionuclide physical decay constant, $\lambda_{\text {phys }}$ was considered known. The parameters of fitting functions were denoted as $C_{k}$ and $\lambda_{k}$.

$$
\begin{aligned}
f_{2}(t) & =C_{1} e^{-\left(\lambda_{1}+\lambda_{\text {phys }}\right) t} & & (K=2) \\
f_{3}(t) & =C_{1} e^{-\left(\lambda_{1}+\lambda_{\text {phys }}\right) t}+C_{2} e^{-\lambda_{\text {phys }} t} & & (K=3) \\
f_{4 a}(t) & =C_{1}\left[e^{-\left(\lambda_{1}+\lambda_{\text {phys }}\right) t}-e^{-\left(\lambda_{2}+\lambda_{\text {phys }}\right) t}\right] & & (K=3) \\
f_{4}(t) & =C_{1} e^{-\left(\lambda_{1}+\lambda_{\text {phys }}\right) t}+C_{2} e^{-\left(\lambda_{2}+\lambda_{\text {phys }}\right) t} & & (K=4)
\end{aligned}
$$

For every voxel $x$, the associated TAC $A(x, t)$ was successively fitted by all models with a nonlinear least square optimization. The Levenberg-Marquardt method was used, together with DenseQR linear solver that uses QR decomposition of the Jacobian and all default parameters of the Ceressolver package. ${ }^{17}$ Before the optimization, the initial fitting parameters were determined with a conventional curve stripping procedure. For every voxel $x$, when the fitting converged, the coefficient of determination $R^{2}$ was used to measure the goodness of fitting, $R^{2}=1-\frac{R S S}{T S S}$, with $R S S=\sum_{l} f\left(t_{l}\right)-A$ $\left.\left(t_{l}\right)\right)^{2}$ the Residual Sum of Squares, TSS $=\sum_{l}\left(A\left(t_{l}\right)-\bar{A}\right)^{2}$ the Total Sum of Squares, $\bar{A}=\frac{1}{N} \sum_{l} A\left(t_{l}\right)$ the mean of the observed data, and $l$ the index of the time-points. If the optimizer did not converge and reached the maximum number of iterations, or if $R^{2}$ was lower than a threshold value, $R_{\min }^{2}$, voxel fitting was considered as failed.

\section{B. Model selection}

For every voxel, after all the fitting processes were performed, a model was selected among the ones that did not fail. This selection was performed with the Akaike information criterion (AIC). This criterion characterizes the information loss when a model is used to approximate the true distribution. The lowest AIC value corresponds to the lower loss. AIC was defined as $A I C=2 K-2 \ln L$ where $K$ is the number of model parameters, and $L$ is the maximum value of the likelihood function. The variance of the model's residuals distributions (RSS) was used as the maximum likelihood. The Akaike criterion hence becomes AIC $=N \ln (R S S /$ $N)+2 K$. It should be noted that for small $N$, the AICc, a second-order bias correction of AIC, should be used (if N-K$1<0$ ) because it should decrease the probability of overfitting. However, for the sake of genericity it has been decided to use AIC rather than AICc throughout. Other model selection methods such as Bayesian information criterion (BIC) could alternatively be used here. We denoted this approach as the voxel-based multimodel fitting method (VoMM). 


\section{C. Integration}

Once the fitting model for a voxel is selected, the TIA could be computed as the area under the curve (AUC), $\tilde{A}=\sum_{k} \frac{C_{k}}{\lambda_{k}+\lambda_{\text {phys }}}$. Organ TIA were computed by integrating activities from the individual voxels belonging to the organ ROI. In order to illustrate differences that may occur between ROI-based and voxel-based approaches, another kinetic parameter of interest was also considered: the mean residence time (MRT). MRT indicates the average amount of time the injected substance stays in the body, which is a useful parameter when studying pharmacokinetics. This quantity must not be confused with the "residence times", now termed timeintegrated activity coefficients ${ }^{2}$ (TIAc), which are the time integrated activities in the source organs per administered activity. The MRT is computed as MRT = AUMC/AUC, with $\mathrm{AUMC}=\sum_{k} \frac{C_{k}}{\left(\lambda_{k}+\lambda_{2}\right)^{2}}$ (the area under the first moment curve) and AUC. Average MRT inside a ROI was obtained by averaging MRT values in the voxels of that ROI.

\section{D. Restricted integration}

An alternative restricted method to estimate TIA was also considered, denoted by SMr (Single Model restricted). Here, only the tail of the TAC was fitted, starting at the point $t_{m}$ where $m$ was the last time-point before the activity decrease. If the TAC do not decrease at the end of the curve, the last three time-points were considered for fitting. The TIA was estimated with trapezoid integration on the first part of the curve, and by monoexponential fitting with $f_{3}$ for the end of the curve. With this method, only TIA was available.

\section{EXPERIMENTS}

\section{A. Notations}

In the following sections, the fitting methods from Table I were studied. The voxel-based fitting method was compared to the standard ROI-based method. The latter averages all voxel values in a given ROI and performs the fitting with the multimodel approach, the best model being chosen with AIC. In all experiments, the number of successful fits, $P_{f i t}$, was determined.

\section{B. Numerical experiment}

The following numerical experiment was designed to serve as a reference and compare reference models to estimated

TABLE I. Names of the different fitting methods.

\begin{tabular}{lll}
\hline \hline voxel-based & SM-f2 & Fitting with model $f_{2}$ (monoexponential) \\
voxel-based & SM-f3 & Fitting with model $f_{3}$ (bi-exponential) \\
voxel-based & SM-f4a & Fitting with model $f_{4 a}$ (bi-exponential) \\
voxel-based & SM-f4 & Fittin with model $f_{4}$ (bi-exponential) \\
voxel-based & VoMM & Multimodels fitting $\left(f_{2}, f_{3}, f_{4 a}, f_{4}\right)$ \\
voxel-based & SM-f3r & Restricted (trapezoid) fitting with model $f_{3}$ \\
roi-based & ROI-based & For TIA and MRT estimation based on ROI \\
\hline \hline
\end{tabular}

models. Several TACs were generated from different pharmacokinetic models $\left(f_{2}, f_{3}, f_{4 a}\right.$ and $\left.f_{4}\right)$. For each, the parameters $C_{k}$ and $\lambda_{k}$ were taken from TAC fitting of ROIs in patient data. ${ }^{14}$ These models, illustrated in Fig. 1, were chosen as they characterize the activity evolution of a monoclonal antibody in the heart $\left(f_{2}\right.$ or $\left.f_{3}\right)$, in the liver $\left(f_{4 a}\right)$ or in a tumor $\left(f_{4}\right)$. To generate a set of different models, the parameters of the models were randomly modified by a uniform distribution around $\pm 50 \%$ (arbitrarily) of their initial values. The models were sampled at six time-points $(\mathrm{T} 0+2 \mathrm{~h},+5 \mathrm{~h},+24 \mathrm{~h},+48 \mathrm{H}$, $+70 \mathrm{H},+144 \mathrm{H}$ ), with a stochastic uniform deviation of $50 \%$ to consider different time-points. Noise in reconstructed images by iterative algorithms is log-normal ${ }^{18}$ distributed, but, for simplicity reasons, we added Poisson noise to the TAC. Several noise levels were considered: the numbers of counts were scaled by a factor from 0.025 to 1.5 , starting from a counts number equal to 2000 counts, thus corresponding to 50 counts to 3000 counts. This range of values was selected because it corresponds to typical values observed in clinical SPECT images. ${ }^{14}$ Poisson noise corresponds to fluctuation around the mean with a standard deviation equal to the square root of the mean, modeled by $I=N\left(I_{0}\right)$, with $I_{0}$ the initial number of counts, and $N\left(I_{0}\right)$ a Poisson-distributed random variable of mean $I_{0}$. The reference TIA values $\tilde{A}_{\text {ref }}$ were computed from the initial models without noise, and compared to the TIA estimated from the noisy TAC denoted by $\tilde{A}_{\text {est }}$. We measured the error as the Root Mean Square of the relative TIAs error [RMSE, Eq. (2)], with $\mathrm{M}$ the number of tests. Several fitting methods were considered: SM-f2, SM-f3, SMf4a, SM-f4, VoMM, SM-f3r. We also computed the number of times the optimizer did not converge. We fixed the maximum number of iteration to 200 for all fitting methods.

$$
\epsilon=\frac{\tilde{A}_{r e f}-\tilde{A}_{e s t}}{\tilde{A}_{r e f}} \quad \text { RMSE }=\sqrt{\frac{\sum_{i=1}^{M} \epsilon_{i}^{2}}{M}}
$$

\section{C. Clinical data}

Clinical images were taken from a phase I radio-immunotherapy clinical trial called Synfrizz. ${ }^{14}$ This trial was conducted with OTSA101 monoclonal antibody (mAb) developed by OncoTherapy Science Inc. that targets a receptor overexpressed in synovial sarcomas, frizzled homolog 10 (FZD10). Each patient benefited from pretherapeutic dosimetry based on six SPECT-CT acquisitions at T0+2h, T0+5h, $\mathrm{T} 0+24 \mathrm{~h}, \mathrm{~T} 0+48 \mathrm{H}, \mathrm{T} 0+72 \mathrm{H}, \mathrm{T} 0+144 \mathrm{H}$, with $\mathrm{T} 0$ the time of ${ }^{111}$ In-labeled monoclonal antibodies injection. Voxel sizes were $4.42^{3} \mathrm{~mm}^{3}$ and $0.98 \times 0.98 \times 1.25 \mathrm{~mm}^{3}$, respectively, for SPECT and CT images. SPECT acquisition time was $30 \mathrm{~min} /$ step, followed by reconstruction using the reconstruction algorithm (OSEM, 10 iterations and 5 subsets) that takes into account attenuation and scatter correction (DEW). We selected a patient from this study with several ROIs (liver, lung, heart, bone marrow from L2-L4, ${ }^{19}$ kidney, spleen, and several lesions). The $\mathrm{CT}$ images were registered as described in Ref. [20] and implemented in Elastix software. ${ }^{21}$ From the 


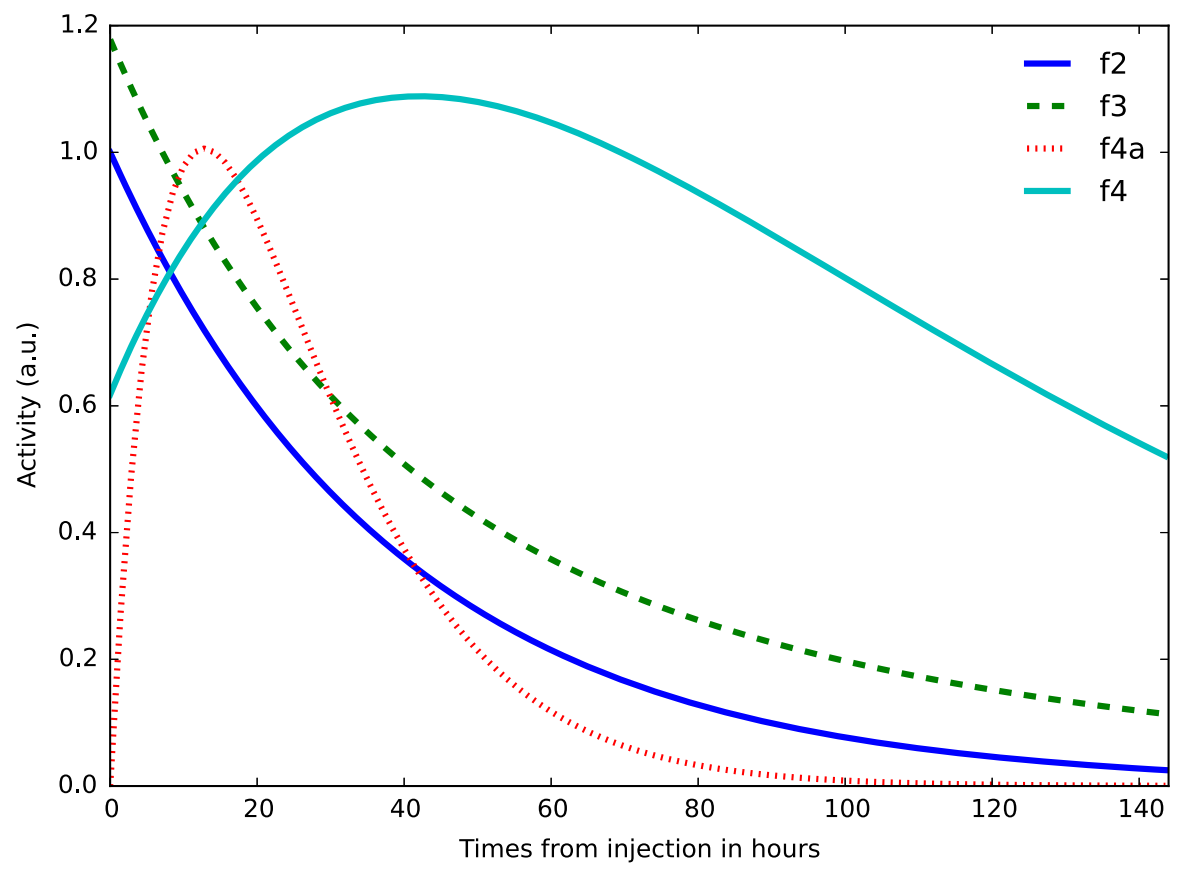

FIG. 1. Illustration of four TAC pharmacokinetic models retrieved from a patient in the Synfrizz clinical trial. ${ }^{14}$ The curves were scaled arbitrarily to fit on the same plot.

resulting Deformable Vector Fields (DVF), all corresponding SPECT images were then warped to the coordinate system of the first CT volume. The uncertainty of the registration was estimated to be less than $2 \mathrm{~mm}$, except for the lower part of lung where respiratory motion leads to additional uncertainty. SPECT images were calibrated, registered and aligned, and converted from ${ }^{111} \mathrm{In}$ to ${ }^{90} \mathrm{Y}$ by decay correction and half-life substitution, assuming a similar biological decay. Voxel values were decay corrected, considering that $A$ $(x, 0)=A(x, t) e^{\lambda t}$, and the values were scaled by $e^{\left(\lambda_{I n} t\right)} e^{\left(-\lambda_{Y} t\right)}$, with $\lambda_{I n}$ and $\lambda_{Y}$ the physical decay constant of respectively ${ }^{111}$ In and ${ }^{90} \mathrm{Y}$. Values at time $=0$ were obtained from the radionuclide supplier. Initial concentrations were given by the radiochemistry laboratory of the hospital. Times at acquisition were obtained from the DICOM tag. Injected quantities were obtained from clinical team, taking the residual activity in the syringe into account. Voxel-based fitting was performed with VoMM, SM-f2, SM-f3, SM-f4, and SM-f3r methods. TIA and MRT were estimated in every ROI with all voxel-based and ROI-based methods, as well as the number of successful fits, $P_{f i t}$.

\section{RESULTS}

Figure 2 shows TIA RMS errors estimated with the selected fitting methods on the numerical test. The percentage of failures, due to either reaching the maximum number of iterations or not reaching the minimum coefficient of determination $R_{\min }^{2}$, is also presented in the bottom figure.

With regard to patient image data, Fig. 3 shows the proportion of successfully fitted voxels with several methods and with several selected $R_{\min }^{2}$ values $(0.6,0.7,0.8,0.9)$ to illustrate the influence of this parameter.
Figure 4 presents TIA estimation in various ROIs with several fitting methods (ROI-based, VoMM, SM-f2, SM-f3, SM-f4, and SM-f3r). For voxel-based estimation, the standard deviation of the voxels mean value in the region is shown as one standard deviation length error bar. TIA values were normalized with respect to the TIA in the liver estimated with the ROI-based method. Similarly, Fig. 5 shows the average MRT (in hours) in ROI computed from several fitting methods. MRT values are compared to the ${ }^{90} \mathrm{Y}$ half-life equal to $64 \mathrm{~h}$.

Figure 6 illustrates two voxel-based TIA coronal slices fitted with SM-f4 (left) and VoMM (right). Yellow (gray) pixels correspond to voxels where fitting failed. Figure 7 shows voxel-based VoMM estimation of MRT. To help visualization, some ROIs (liver, spleen, and lesion) are delineated. The bottom right image depicts the selected model for every voxel.

The computation time depends on several factors: the number of voxels, the number of models under test and the noise level that directly impacts the convergence of the fitting procedures. The implementation was performed in $\mathrm{C}++$, with Ceres-solver ${ }^{17}$ as fitting algorithm and ITK, ${ }^{22}$ without any particular optimization. On a single core Intel Xeon CPU E5$16603.3 \mathrm{GHz}$, the order of magnitude of the computation speed is 800 voxels per second (50,000 voxels per minute) for five models. The computation time for real patient data (577,476 voxels) took about 10 min with a single core, however, it could be further parallelized.

\section{DISCUSSION}

It can be seen in Fig. 2 that RMS error tends to decrease for large number of counts, that is, for a lower noise level. 

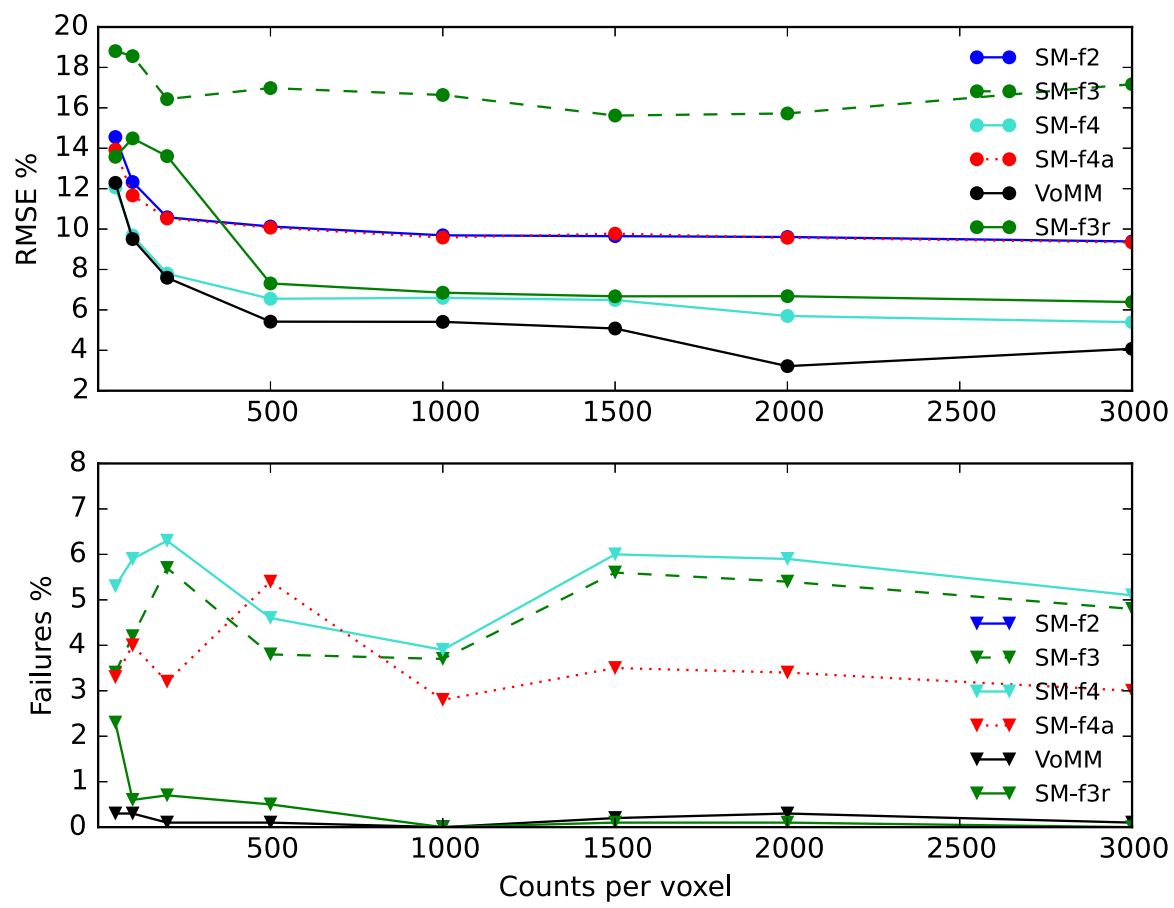

FIG. 2. (Top) RMS errors for several fitting methods (VoMM, SM-f2, SM-f3, SM-f4 and SM-f3r) as a function of number of counts per voxel. (Bottom) percentage of fitting failures as a function of number of counts per voxel. SM-f2 is not presented as failure rates were higher than $25 \%$.

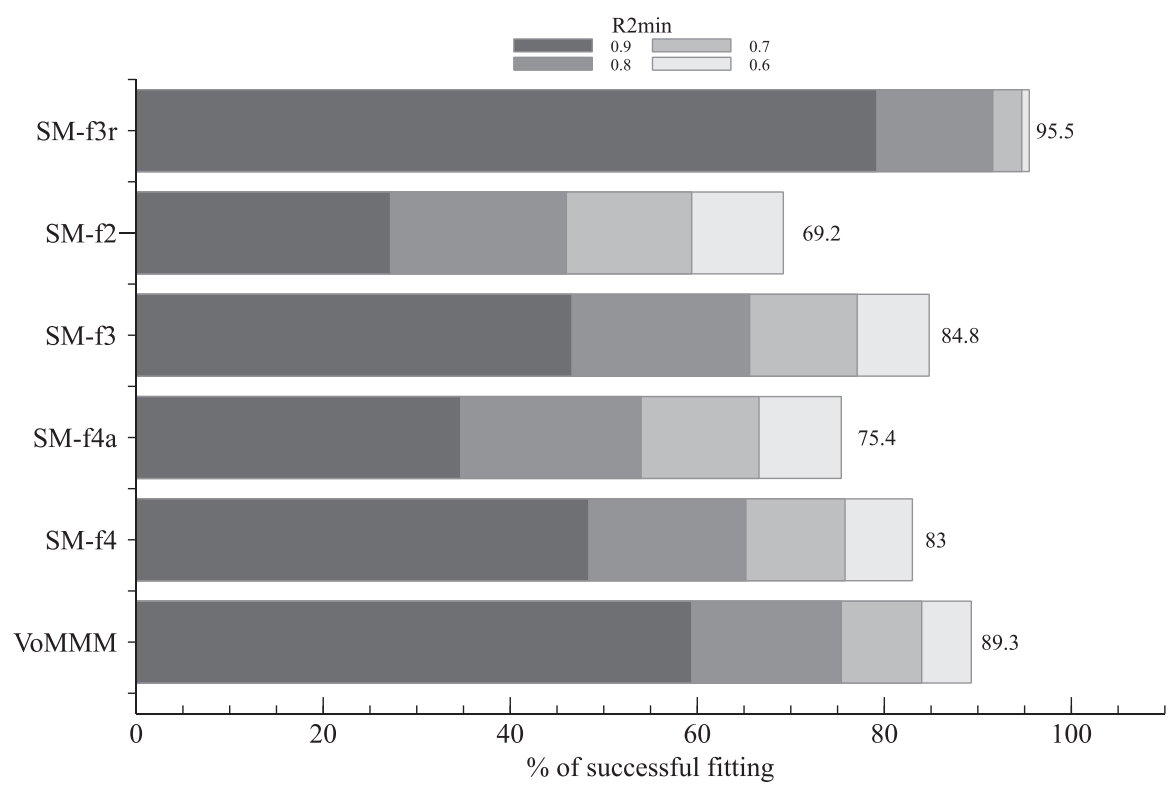

FIG. 3. Percentage of successful voxels fitting for several methods and several $R_{\min }^{2}$ values on patient data.

VoMM method leads to a lower error, around $6 \%$ for voxels with 1000 counts, except for higher noise level where SM-f4 is slightly better. In those cases, it means that VoMM method sometimes selected a model with a slightly larger error than $f_{4}$, but with a better AIC criterion that favor a lower number of parameters. VoMM, SM-f4, and SM-f3r depicted close results, while other methods show higher errors. This behavior was expected: the model with the highest number of parameters, SM-f4, is better than the others. Restricted methods lead to good results, close to SM-f4 when the noise is sufficiently low (more than 500 counts). Failure rate is very low, lower than $1 \%$ for VoMM and SM-f3r, while all other methods lead to larger rates, around 5\%. No clear trends could be observed according to the noise level. The observed behavior validates the principle of the model selection mechanism: good RMSE could be obtained with $f_{4}$ model having four free parameters, but VoMM method allows slightly better results while keeping a very low failure rate. We also 


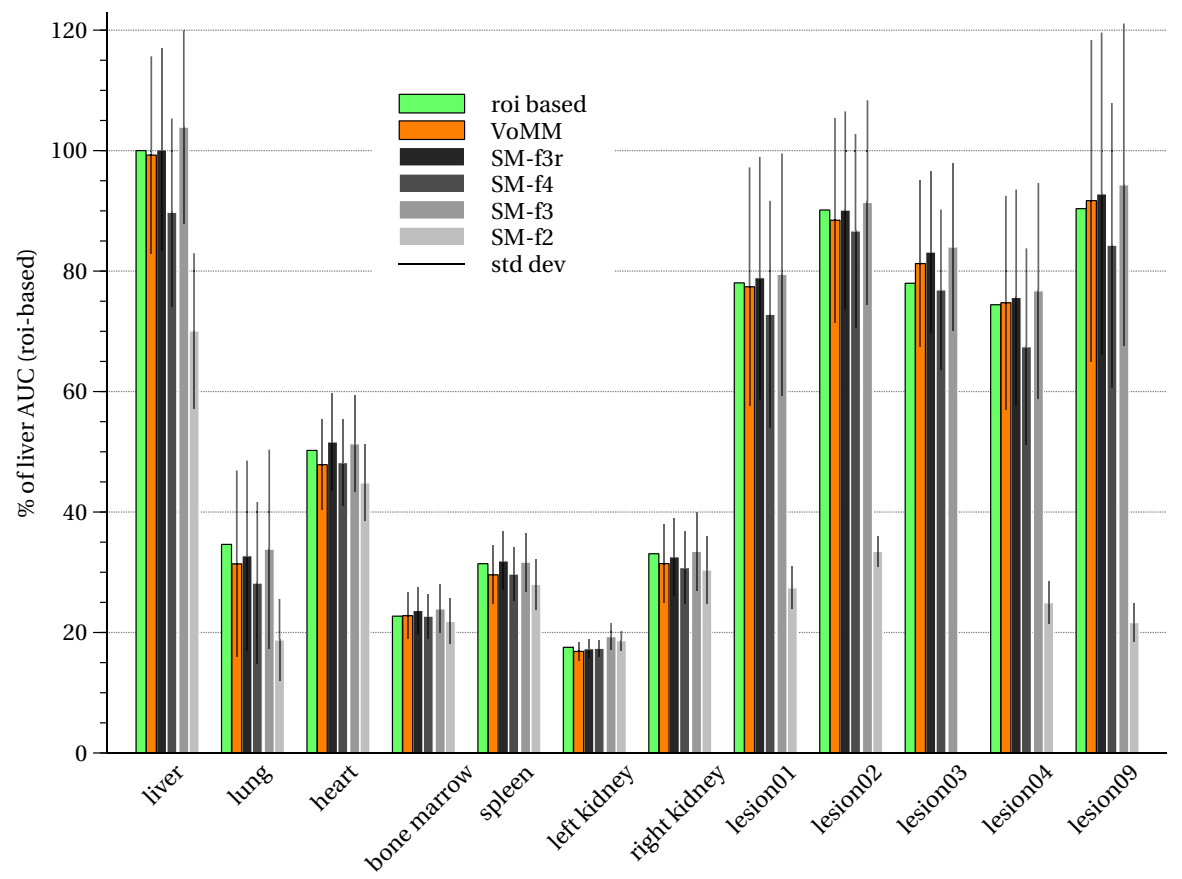

FIG. 4. Time integrated activities for several ROIs estimated with various fitting methods. For voxel-based methods, standard deviation among all voxels in the ROI is depicted with one standard deviation length vertical bar.

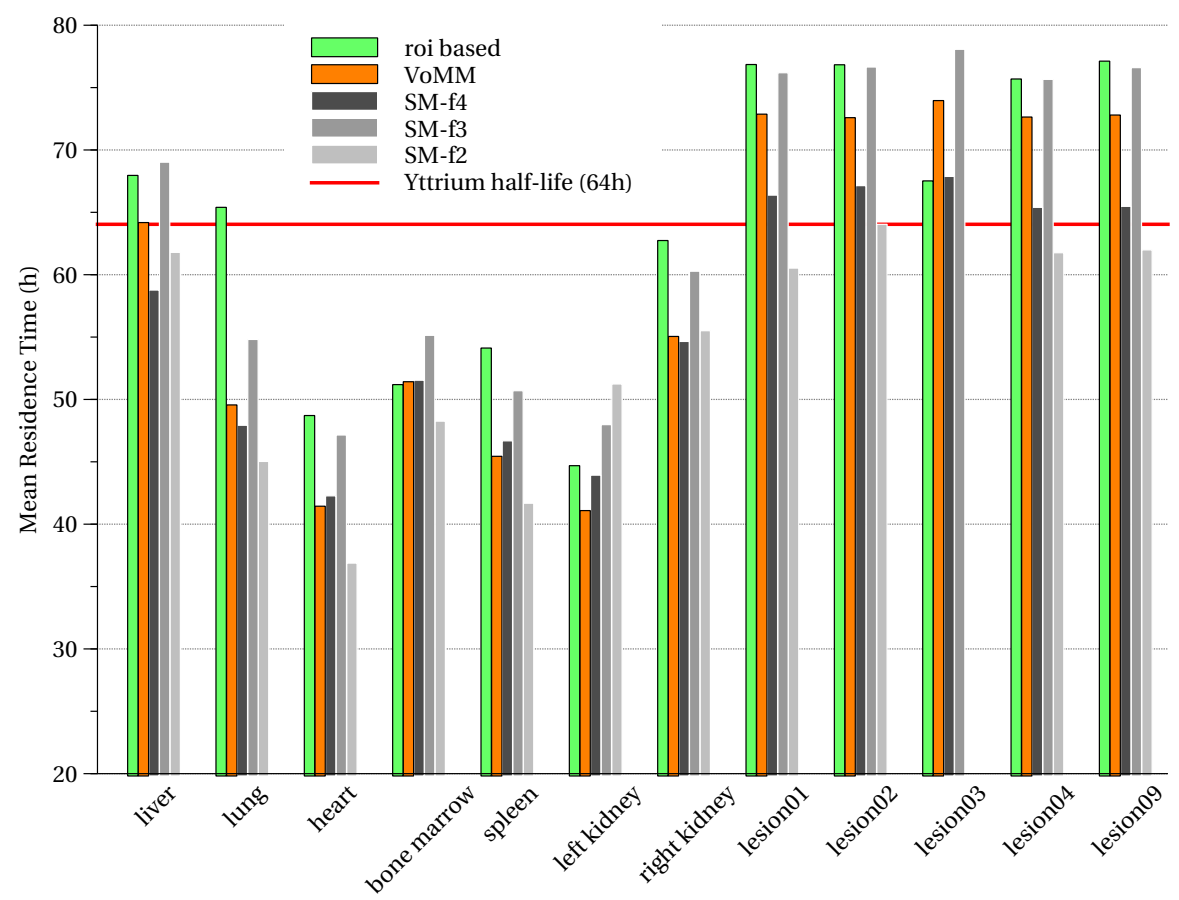

FIG. 5. Mean residence time (MRT) in hours for several ROIs, estimated with various fitting methods.

observed that the starting values of the parameters before Levenberg-Marquardt optimization plays a crucial role in the rate of convergence. Compared to the method proposed in Jackson et al. study, ${ }^{5}$ no additional weight to final points were used, no preconditioning. One additional advantage of using VoMM is to limit overfitting. This is the principle of parsimony that privileges models with a lower number of parameters thanks to the AIC. One can conclude from this experiment that VoMM is feasible, leads to lower error, and to more robust TIA estimation than other methods.

With regard to clinical data, we observed in Fig. 3 that the rate of successfully fitted voxels, $P_{f i t}$, increased when $R_{\min }^{2}$ decreased. $P_{f i t}$ values were lower for single model fitting methods compared to VoMM. Restricted trapezoid approach 

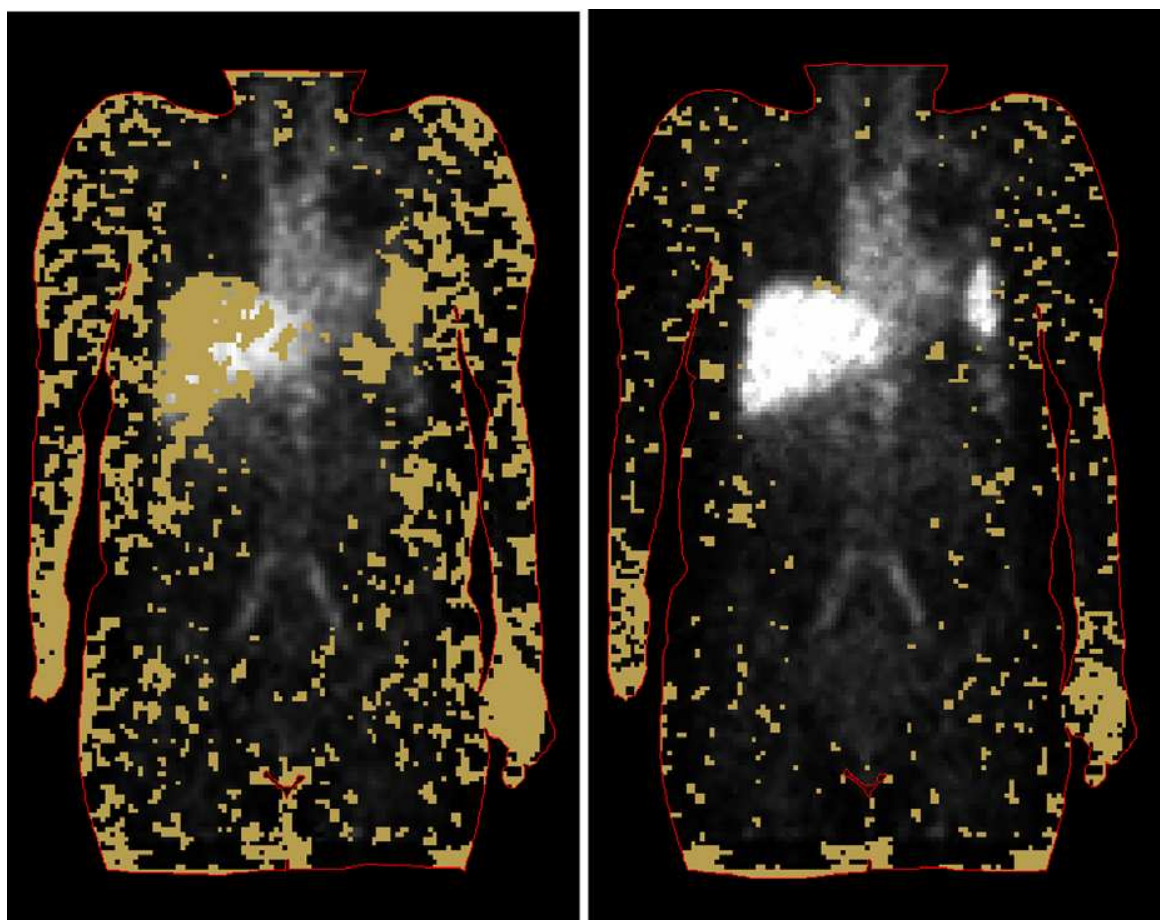

FIG. 6. Distribution of TIA estimated with SM-f4 (left) and VoMM (right) methods on a coronal slice. Yellow pixels correspond to voxels that cannot be fitted.
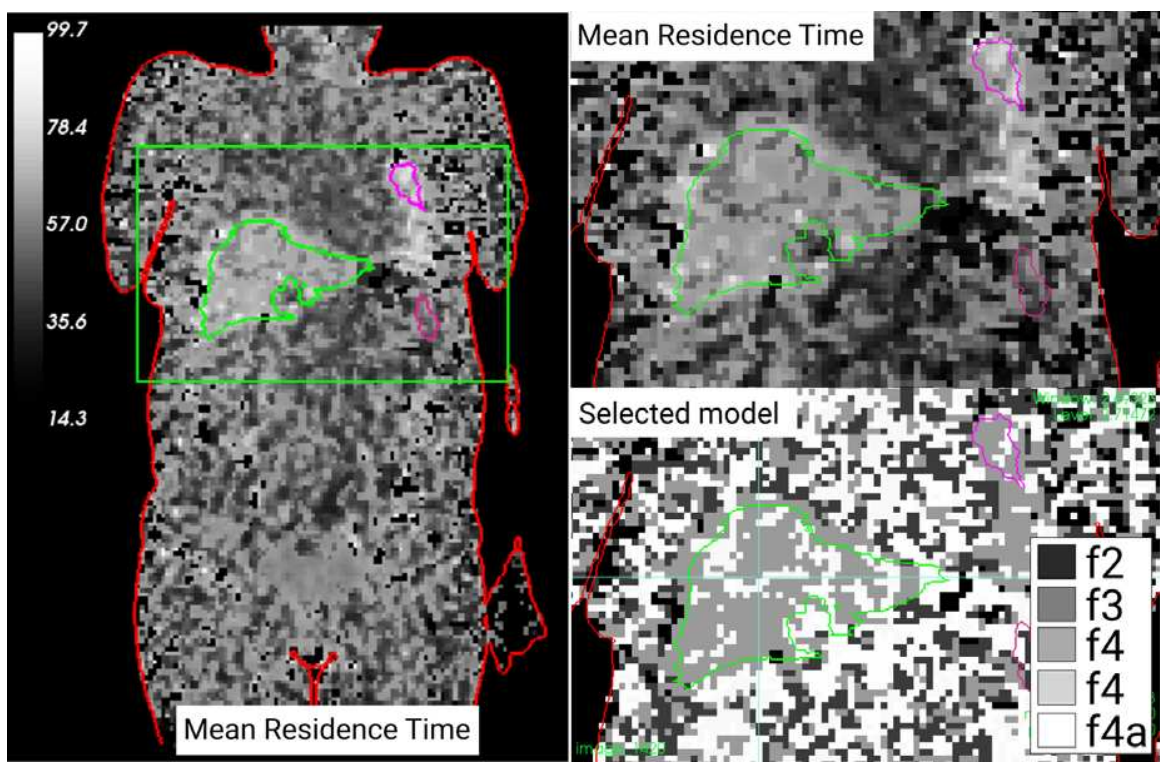

FIG. 7. Distribution of MRT computed with VoMM methods, gray values scale corresponds to the MRT values (left and top right images). Right images are a closeup of the rectangle on the left. Bottom right image shows the corresponding selected models in each voxel.

SM-f3r leads to higher success rates, which was also expected as the fitting is only performed for the final part of the TAC. With $R_{\min }^{2}=0.6$, more than $89 \%$ of the voxels may be fitted with VoMM, which corresponds to more than $95 \%$ of the total activity. About $60 \%$ gave $R^{2}$ greater than 0.9 . In practical situations, the remaining failed voxels may, for example, be inferred from neighboring voxels assuming a local continuity. Another approach could be used to decrease image noise by applying preprocessing such as Gaussian filtering. We did not investigate further this point. We however conclude that, on realistic clinical data, voxel-based TAC fitting modeling is feasible.

From Fig. 4, we observed that the TIA estimation differences between methods were relatively limited, below $10 \%$, except for SM-f2 (monoexponential model) which is clearly insufficient to obtain estimation close to the ROI-based 
method. Hence, voxel-based methods to estimate mean TIA within a ROI is probably not necessary, the difference with ROI-based estimation being low. However, voxel-based estimation brings more insight as it allows the characterization of heterogeneity within an ROI. The spatial heterogeneity within the ROI was relatively high in some ROI. For example, with VoMM, the standard deviation of TIA values within ROI was $\mathrm{SD} \approx 16 \%$ for liver and lung ROI, and could reach $\mathrm{SD} \approx 25 \%$ within a lesion. On the contrary, bone marrow region has less than $4 \%$ variation. Figure 5 depicts MRT estimation and illustrates that differences between voxel and ROI-based estimations could be large. In the liver region the MRT estimation was close to the half-life of the ${ }^{90} \mathrm{Y}(64 \mathrm{~h})$, and even larger for lesions. It could be explained by either noise in the data and/or a late mAb uptake and accumulation. For lung ROI, MRT is overestimated with the ROI-based method compared to voxel-based approaches. The TIA and MRT spatial distributions are illustrated in Figs. 6 and 7 where the heterogeneity within ROIs can be observed. Voxelbased modeling could serve as input for automated classification methods. Every fitting was performed with mono- and biexponential models, but, in principle, any other set of models could be used.

Several limitations occur for voxel-based TAC analysis. First, the uncertainty of images registration affects TAC analysis, even if the registration uncertainty is lower (around $2 \mathrm{~mm}$ ) than SPECT resolution $(4 \mathrm{~mm})$. The main impact is probably due to respiratory motion. Indeed, SPECT acquisition is a slow process according to breathing cycle, leading to blurred activities near moving areas such as the diaphragm. CT acquisitions are faster and images may depict artifacts, leading to erroneous deformation estimation. Voxel-based analysis near those moving areas is thus subject to caution. Moreover, SPECT statistical noise and Partial Volume Effect (PVE) is another source of uncertainty. Even if some correction techniques has been proposed, ${ }^{23} \mathrm{PVE}$ is still largely unsolved. Quantitation of the impact of those types of uncertainty on voxel-based TAC analysis could be the subject of another study.

\section{CONCLUSION}

We proposed a voxel-based multimodel fitting method (VoMM) that automatically selects the most appropriate fitting function to model a continuous time activity curve on a per voxel basis. To our knowledge this is the first time a multimodel per-voxel fitting method has been proposed and studied. VoMM method is independent of the radionuclide and converges for about $90 \%$ of voxels in the tested patient images (and for more than 95\% of the total activity). We showed that if the goal is to estimate TIA per ROI only, conventional ROI-based method leads to results close to VoMM and is much faster. Also, if the goal is to estimate voxel-based TIA only, the restricted trapezoid method SM-f3r may be used alternatively to VoMM thanks to the small differences. However, we observed non-negligible differences between ROI-based and voxel-based MRT estimation, that could reach $25 \%$. For pharmacokinetic analysis, only voxel-based methods allow to take into account the spatial heterogeneity and the nonuniformity of the activity distribution within an ROI. In these situations, the use of VoMM is meaningful as it also prevents model overfitting thanks to AIC. As perspective, voxel-based analysis of the fitting models may be helpful for segmentation of the image into components with similar pharmacokinetic behavior. The method could also be applied to other images than SPECT.

\section{ACKNOWLEDGMENTS}

This work was partly supported by t-Gate ANR-14CE23-0008, the SIRIC LYric Grant INCa-DGOS-4664, and the LABEX PRIMES (ANR-11-LABX-0063/ ANR-11IDEX-0007). The authors thank OK and RB for helpful proofreading.

\footnotetext{
a) Author to whom correspondence should be addressed. Electronic mail: david.sarrut@ creatis.insa-lyon.fr.
}

\section{REFERENCES}

1. Dewaraja YK, Schipper MJ, Shen J, et al. Tumor-absorbed dose predicts progression-free survival following (131)i-tositumomab radioimmunotherapy. J Nucl Med. 2014;55:1047-1053.

2. Bolch WE, Eckerman KF, Sgouros G, Thomas SR. Mird pamphlet no. 21: a generalized schema for radiopharmaceutical dosimetry-standardization of nomenclature. J Nucl Med 2009;50:477-484.

3. Lin H, Jing J, Cai J, Xu L. A voxel-dose algorithm of heterogeneous activity distribution for monte-carlo simulation of radionuclide therapy dosimetry. Cancer Biother Radiopharm. 2012;27:344-352.

4. Green A, Flynn A, Pedley RB, Dearling J, Begent R. Nonuniform absorbed dose distribution in the kidney: the influence of organ architecture. Cancer Biother Radiopharm. 2004;19:371-377.

5. Jackson PA, Beauregard J-M, Hofman MS, Kron T, Hogg A, Hicks RJ. An automated voxelized dosimetry tool for radionuclide therapy based on serial quantitative spect/CT imaging. Med Phys. 2013;40: 112503.

6. Kost SD, Dewaraja YK, Abramson RG, Stabin MG. Vida: a voxel-based dosimetry method for targeted radionuclide therapy using geant4. Cancer Biother Radiopharm. 2015;30:16-26.

7. Grassi E, Fioroni F, Ferri V, et al. Quantitative comparison between the commercial software stratos $\left({ }^{\circledR}\right)$ by philips and a homemade software for voxel-dosimetry in radiopeptide therapy. Phys Med. 2015;31:72-79.

8. Bolch WE, Bouchet LG, Robertson JS, et al. Mird pamphlet no. 17: the dosimetry of nonuniform activity distributions-radionuclide $\mathrm{s}$ values at the voxel level. Medical internal radiation dose committee. J Nucl Med. 1999;40:11S-36S.

9. Hippeläinen E, Tenhunen M, Sohlberg A. Fast voxel-level dosimetry for (177)lu labelled peptide treatments. Phys Med Biol. 2015;60: 6685-6700.

10. Pacilio M, Amato E, Lanconelli N, et al. Differences in 3D dose distributions due to calculation method of voxel S-values and the influence of image blurring in SPECT. Phys Med Biol. 2015;60:1945-1964.

11. Ljungberg M, Celler A, Konijnenberg MW, et al. Mird pamphlet no. 26: Joint eanm/mird guidelines for quantitative 177lu spect applied for dosimetry of radiopharmaceutical therapy. J Nucl Med. 2016;57:151-162.

12. Sjogreen-Gleisner K, Rueckert D, Ljungberg M. Registration of serial spect/CT images for three-dimensional dosimetry in radionuclide therapy. Phys Med Biol. 2009;54:6181-6200.

13. Ao ECI, Wu N-Y, Wang S-J, Song N, Mok GSP. Improved dosimetry for targeted radionuclide therapy using nonrigid registration on sequential spect images. Med Phys. 2015;42:1060-1070.

14. Sarrut D, Badel J-N, Halty A, et al. 3d absorbed dose distribution estimated by monte carlo simulation in radionuclide therapy with a 
monoclonal antibody targeting synovial sarcoma. EJNMMI Phys. 2017;4:6

15. Kletting P, Schimmel S, Kestler HA, et al. Molecular radiotherapy: the nukfit software for calculating the time-integrated activity coefficient. Med Phys. 2013;40:102504.

16. Glatting G, Kletting P, Reske SN, Hohl K, Ring C. Choosing the optimal fit function: comparison of the akaike information criterion and the $\mathrm{f}$ test. Med Phys. 2007;34:4285-4292.

17. Agarwal S, Mierle K, et al. Ceres solver. http://ceres-solver.org.

18. Barrett HH, Wilson DW, Tsui BM. Noise properties of the em algorithm: I. Theory. Phys Med Biol. 1994;39:833-846.

19. Ferrer L, Kraeber-Bodéré F, Bodet-Milin C, et al. Three methods assessing red marrow dosimetry in lymphoma patients treated with radioimmunotherapy. Cancer. 2010;116:1093-1100.
20. Delmon V, Rit S, Pinho R, Sarrut D. Registration of sliding objects using direction dependent b-splines decomposition. Phys Med Biol. 2013;58:1303-1314.

21. Klein S, Staring M, Murphy K, Viergever MA, Pluim JPW. Elastix: a toolbox for intensity-based medical image registration. IEEE Trans Med Imaging. 2010;29:196-205.

22. Johnson HJ, McCormick MM, Ibanez L. The ITK Software Guide: Design and Functionality. Kitware Inc.; 2015. https:/itk.org/Wiki/ITK/ FAQ\#How_do_I_cite_the_use_of_ITK_in_a_publication.3F.

23. Erlandsson K, Buvat I, Pretorius PH, Thomas BA, Hutton BF. A review of partial volume correction techniques for emission tomography and their applications in neurology, cardiology and oncology. Phys Med Biol. 2012;57:R119-R159. 